\title{
ERRATA, VOLUME 50
}

$\mathrm{J}$. Hadamard, $A$ known problem of geometry and its cases of indetermination.

p. 520. Footnote 1 should read "Mathematicae Notae, Boletin del Instituto de Matematica vol. 3 (1945) p. 155, Rosario (Argentine Republic)."

\section{ERRATA, VOLUME 51}

D. H. Hyers, Linear topological spaces.

p. 9, line 1. For "neighborhood system $U$ " read "neighborhood system V."

p. 10 , line 17. For "point $y \neq x$ " read "point $y$ of $S$, with $y \neq x$."

p. 11 , line 28 . For "set $D$ " read "set $E$."

p. 14, line 20. To conditions (2), (a) and (b), of Definition 6.1 add the condition:

"(c) $\epsilon\left(x_{0}, x_{1}, x_{2}\right)$ is continuous in the pair $\left(x_{1}, x_{2}\right)$ at the point $\left(\theta, x_{2}\right)$ for each $x_{2}$ in $L_{1} ;$;".

p. 14 , line 36 . For " $\epsilon(\theta, y, z)$ " read " $\epsilon_{r}(\theta, y, z)$."

p. 15, last line. For " $m$-differential" read " $M$-differential."

H. S. Wall, Note on the expansion of a power series into a continued fraction.

p. 98, line 5. Instead of

read

$$
\text { “ } \frac{A_{n+1}(z)}{B_{n+1}(z)}-\frac{A_{n}(z)}{B_{n}(z)}=\frac{a_{0} a_{1} \cdots a_{n}}{z^{2 n+1}}+\frac{h_{n}}{z^{2 n+2}}+\cdots ”
$$

$$
\text { “ } \frac{A_{n+1}(z)}{B_{n+1}(z)} B_{n}(z)-A_{n}(z)=\frac{a_{0} a_{1} \cdots a_{n}}{z^{n+1}}+\frac{h_{n}}{z^{n+2}}+\cdots .
$$

H. E. Salzer, Formulas for direct and inverse interpolation of a complex function tabulated along equidistant circular arcs, abstract 51-7127.

p. 522, line 12 of abstract. For " $\sum C_{k}^{(n)}\left(p_{m}+i q_{m}\right)$ " read " $\sum C_{\boldsymbol{k}}^{(m)}\left(p_{m}+i q_{m}\right) . "$

H. Bateman, The control of an elastic fluid.

p. 629 , line 4 . The footnote symbol should be 79 instead of 69 .

p. 645 , lines 4 and 5 . For "equation we form (A) the function" read "equation (A) we form the function."

Isaac Opatowski, Direct and reverse transitions in Markoff chains, abstract 51-9-184.

p. 665 , line 6 of abstract. Instead of " $k_{1}$ " read " $k_{i .}$ " Instead of " $g_{1}$ " read " $g_{i} . "$ 